\title{
Treatment of Cavernous Sinus Aneurysms with Flow Diversion: Results in 44 Patients
}

\author{
R.C. Puffer, M. Piano, G. Lanzino, L. Valvassori, D.F. Kallmes, L. Quilici, H.J. Cloft, and E. Boccardi
}

\begin{abstract}
BACKGROUND AND PURPOSE: Aneurysms of the cavernous segment of the ICA are difficult to treat with standard endovascular techniques, and ICA sacrifice achieves a high rate of occlusion but carries an elevated level of surgical complications and risk of de novo aneurysm formation. We report rates of occlusion and treatment-related data in 44 patients with cavernous sinus aneurysms treated with flow diversion.
\end{abstract}

MATERIALS AND METHODS: Patients with cavernous segment aneurysms treated with flow diversion were selected from a prospectively maintained data base of patients from 2009 to the present. Demographic information, treatment indications, number/type of flow diverters placed, outcome, complications (technical or clinical), and clinical/imaging follow-up data were analyzed.

RESULTS: We identified 44 patients (37 females, 7 males) who had a flow diverter placed for treatment of a cavernous ICA aneurysm (mean age, 57.2; mean aneurysm size, $20.9 \mathrm{~mm}$ ). The mean number of devices placed per patient was 2.2. At final angiographic follow-up (mean, 10.9 months), $71 \%$ had complete occlusion, and of those with incomplete occlusion, $40 \%$ had minimal remnants ( $<3 \mathrm{~mm}$ ). In symptomatic patients, complete resolution or significant improvement in symptoms was noted in $90 \%$ at follow-up. Technical complications (which included, among others, vessel perforation in 4 patients, groin hematoma in 2, and asymptomatic carotid occlusion in 1) occurred in approximately $36 \%$ of patients but did not result in any clinical sequelae immediately or at follow-up.

CONCLUSIONS: Our series of flow-diversion treatments achieved markedly greater rates of complete occlusion than coiling, with a safety profile that compares favorably with that of carotid sacrifice.

ABBREVIATION: PED = Pipeline Embolization Device

A neurysms originating from the cavernous segment of the internal carotid artery are thought to have a more benign natural history than intracranial aneurysms in other vascular territories, with a substantially lower risk of rupture. ${ }^{1-3}$ Treatment is indicated when these aneurysms are symptomatic and/or reach very large and giant size. Due to the dysplastic nature of these aneurysms and typically difficult morphology, achieving complete aneurysm occlusion by using endovascular "reconstructive" techniques is difficult. "Deconstructive" ICA sacrifice for cavernous ICA aneurysms with or without bypass achieves remarkably high rates of complete aneurysm occlusion. ${ }^{4}$ However, ICA sac-

Received June 17, 2013; accepted after revision September 26.

From the Departments of Neurosurgery (R.C.P.) and Radiology (G.L., D.F.K., H.J.C.), Mayo Clinic, Rochester, Minnesota; and Division of Neuroradiology (M.P., L.V., L.Q., E.B.), Ospedale Niguarda, Milano, Italy.

Please address correspondence to Giuseppe Lanzino, MD, Department of Neurosurgery, Mayo Clinic, 200 1st St SW, Rochester, MN 55905; e-mail: lanzino. giuseppe@mayo.edu

$\equiv$ Indicates article with supplemental on-line tables.

http://dx.doi.org/10.3174/ajnr.A3826 rifice is associated with several potential disadvantages because many of these patients have contralateral mirror ICA aneurysms and ipsilateral ICA occlusion limits therapeutic options for those contralateral aneurysms in the future. Concerns have been raised about long-term hemodynamic effects of ICA sacrifice in young patients, with resultant de novo aneurysm formation in $4 \%-11 \%$ of patients thought to result from increased flow in the anterior and posterior communicating artery regions. ${ }^{5-8}$ Finally, the need for either low- or high-flow bypass in patients who do not tolerate balloon test occlusion may add risk to the overall procedure.

Endoluminal flow-diversion devices have been associated with high rates of complete aneurysm occlusion, even in giant aneurysms. ${ }^{9}$ Cavernous ICA aneurysms appear well-suited to treatment by flow diversion, given the sidewall morphology and lack of critical perforating side branches of the aneurysm in the region of the aneurysm cavity. However, given the relatively benign natural history of these aneurysms and the excellent results reported with ICA sacrifice, it remains critical to assess the risk-benefit ratio for treatment by flow diversion compared with alternative approaches. While there are multiple studies demonstrating a single 
institutional experience treating intracranial aneurysms with flow diverters, to our knowledge, there has been no previous case series published focusing specifically on treatment of cavernous ICA aneurysms by flow diversion. In the current study, we detail the safety and efficacy of flow diversion in 44 patients with cavernous ICA aneurysms.

\section{MATERIALS AND METHODS}

This study was approved by the institutional review board at both institutions where data were collected. From prospectively maintained data bases of patients treated with flow diverters, those who underwent treatment for a cavernous ICA aneurysm from 2009 to the present were selected and retrospectively reviewed. Some of the patients reported were enrolled in the Pipeline for Uncoilable or Failed Aneurysms Study ${ }^{9}$ or were reported as part of a large series on flow diversion. ${ }^{10}$ Demographic information, indication for treatment, type and number of flow diverters placed, treatment outcome, complications (technical or clinical, with 1-sided confidence intervals), and clinical/imaging follow-up data were collected and analyzed. The 5 main operators performing these procedures each had at least 10 years of independent practice, and many of the procedures were performed with 2 experienced operators present.

All patients undergoing treatment were premedicated with aspirin and clopidogrel, and full anticoagulation was maintained during the procedure (target activated clotting time between 250 and 300 seconds). Following the procedure, patients were maintained on dual antiplatelet therapy for 3 months (institution 1) and for 1 month (institution 2). After these time intervals, clopidogrel was discontinued and aspirin was continued indefinitely (institution 1) and for a total of 3 months (institution 2). No patient underwent testing for clopidogrel response. The shorter duration of the dual antiplatelet therapy is related to our belief that if flow diverters are adequately sized, they oppose better against the vessel wall; therefore, after an initial period of potential higher thrombogenicity, the risk of late thromboembolic complications when these devices are used in the proximal ICA is very low and is outweighed by the risk of continuing dual antiplatelet therapy akin to a proximal carotid stent. ${ }^{11}$

All procedures were performed with the patient under general endotracheal anesthesia. Patients were treated with a Pipeline Embolization Device (PED; Covidien, Irvine, California), a Silk flow-diverting stent (Balt Extrusion, Montmorency, France), a Surpass Stent (Stryker Neurovascular, Fremont, California), or, in some cases, a combination of 2 separate devices. There were 2 patients in the series in whom a Liberté stent (Boston Scientific, Natick, Massachusetts) was used to correct a persistent stenosis related to compression from the aneurysm mass; in 1 patient, a LEO stent (Balt Extrusion) was used as a scaffold for the flow diverter to bridge a wide aneurysm neck. Flow diverters were sized to match the maximum diameter of the target vessel, and 1 or multiple devices were used at the discretion of the operators to maximize the chance of complete aneurysm occlusion and/or to ensure adequate coverage of the aneurysm neck and of a segment of the parent artery proximal and distal to it. Digital subtraction angiography was performed at 2 frames per second before and following placement of the flow diverter. Flow diverters were placed under fluoroscopy with a frame rate of 12.5 frames per second.

\section{RESULTS}

We identified 44 patients ( 37 females and 7 males) who had a flow diverter placed for treatment of a cavernous ICA aneurysm. Their clinical and demographic information is included in On-line Table 1 . The mean age for the cohort was $57.2 \pm 14.3$ years (range, $17-81$ years), and the mean aneurysm size was $20.9 \pm 6.9 \mathrm{~mm}$ (range, $4-40$ ). Of note, $48 \%$ of patients (21/44) in this series had giant cavernous sinus aneurysms $(>25 \mathrm{~mm})$. The most frequent presenting symptoms included cavernous cranial neuropathy/ diplopia in 52\% (23/44), headache/retro-orbital pain in 30\% (13/ $44)$, and incidental discovery after an unrelated symptom with or without interval growth in 23\% (10/44). Adjacent aneurysms were present in $27 \%$ of patients (12/44), and in 4 of the patients with adjacent aneurysms, the flow diverter also covered the neck of the adjacent aneurysm, resulting in treatment of multiple aneurysms simultaneously.

Aneurysms were previously treated with coils in only 1 patient. The PED was the only flow diverter used in $64 \%$ of patients (27/ 42), $24 \%$ (10/42) were treated with the Silk device only, 2\% (1/42) were treated with the Surpass flow diverter, and $4 / 42$ patients $(10 \%)$ were treated with a combination of flow diverters and scaffold stents placed telescopically. Multiple devices were required in the initial procedure in $48 \%$ (20/42) of patients to achieve adequate coverage of the aneurysm neck. The mean number of flow diverters placed in this cohort was $2.2 \pm 2.0$ (range, 1-10).

Adequate angiographic and clinical follow-up was available in 36 patients, and their angiographic and clinical outcomes are demonstrated in On-line Table 2. One patient was excluded from occlusion analysis due to subsequent transvenous coiling of the cavernous sinus for treatment of a carotid cavernous fistula. At final angiographic follow-up (3-36 months; mean, 10.9 months), $71 \%(25 / 35)$ had complete occlusion, and of the 10 patients with incomplete occlusion, 40\% (4/10) had minimal remnants present on angiography. In patients who had incomplete occlusion noted on 6-month angiograms and also had a follow-up angiogram at 12 months or later, $50 \%(4 / 8)$ were found to have progressed to complete occlusion at final follow-up (Fig 1).

There were 29 patients who were symptomatic at presentation and had adequate clinical follow-up. Complete resolution or significant improvement of their presenting symptoms was noted in $90 \%$ of patients $(26 / 29)$. Persistent, pre-existing symptoms included continued cranial neuropathy in 2 patients and minor headache in 1 patient. New clinical symptoms developed in $23 \%$ $(10 / 29)$ of patients periprocedurally, mostly $2-5$ days after the treatment, and included localized retro-orbital pain in 5, worsening of pre-existing diplopia in 3 , chemosis in 2 , and a complete cavernous sinus syndrome in 1 patient with a giant cavernous aneurysm. This periprocedural pain resolved completely within the first month in all these patients with medical treatment (pain medications and/or short steroid taper). The patients with worsening or new-onset diplopia experienced resolution of their clinical symptoms by the time of the first clinical follow-up (usually 1 month after treatment), and the patient with a delayed, complete cavernous sinus syndrome had almost complete resolution of 

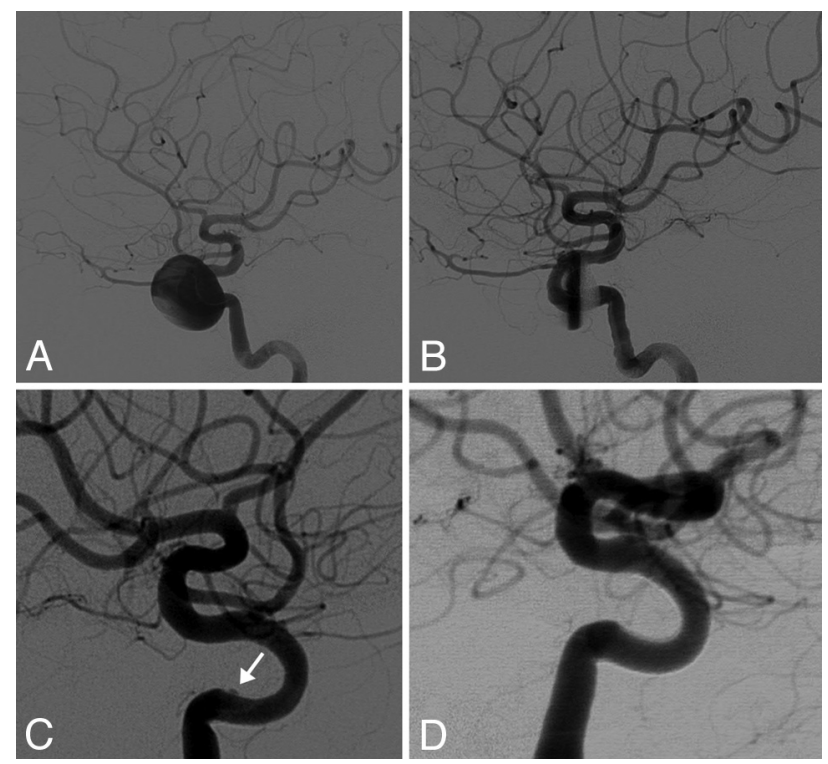

FIG 1. A, Lateral digital subtraction angiography of the left ICA in a 67-year-old woman demonstrates a large $(20 \mathrm{~mm})$ aneurysm originating from the cavernous segment of the ICA. $B$, Immediate postplacement image of 3 PEDs demonstrates stasis of contrast within the aneurysm. C, Follow-up lateral DSA at 6 months demonstrates a minimal remnant (white arrow). D, Final angiographic follow-up demonstrates complete aneurysm occlusion at 36 months.

these new symptoms at 6-month follow-up but continues to have slight upgaze limitation. A small, asymptomatic distal hematoma in the frontal lobe was identified on postprocedural CT in 1 patient $(2 \%, 1 / 42)$.

Intraprocedural technical complications were encountered in $36 \%$ of procedures $(16 / 44)$, including minor catheter-induced vasospasm $(4.5 \%, 2 / 44)$, incomplete opening of the device requiring angioplasty $(16 \%, 7 / 44)$, inability to pass the catheter across the aneurysm due to difficult anatomy $(2 \%, 1 / 44)$, and vessel perforation $(9 \%, 4 / 44)$. Access complications (groin hematoma requiring repair by vascular surgery) occurred in 2 patients $(4.5 \%)$. Delayed, in-stent stenosis of $\geq 50 \%$ was found in 1 patient $(2 \%)$ at follow-up, and in this patient, a Liberté stent was later placed to prevent progression of the stenosis. Asymptomatic ICA occlusion was found in 1 patient (2\%) at follow-up angiography. No patients who experienced intraprocedural or delayed complications had any clinical sequelae either immediately postprocedure or at follow-up (95\% confidence interval, 0\%-9.6\%).

\section{DISCUSSION}

In this study, we demonstrated that flow diverters are an effective treatment technique for cavernous aneurysms, not only for achieving aneurysm closure but also for resolution of presenting clinical symptoms. Remarkably high rates of complete or nearcomplete occlusion were achieved (83\%), even though the mean aneurysm size was approximately $21 \mathrm{~mm}$ and almost $50 \%$ of the cohort had giant aneurysms. Furthermore, $>90 \%$ of cranial neuropathies, either pre-existing or developing soon after treatment, resolved with time in all except 1 patient. These findings are similar to those in a recently published multicenter study on flowdiversion with a subset analysis of 26 cavernous sinus aneurysms. In that study, the authors achieved a complete or near-complete occlusion rate of $100 \%$, with resolution or significant improvement in symptoms in $72 \%$ of patients. ${ }^{12}$ Symptom resolution in these patients was related to decreased pulsation of the aneurysm and to regression of the sac, which follows successful exclusion of the aneurysm with flow diversion as eloquently demonstrated by Szikora et al. ${ }^{13}$ Perianeurysmal inflammation can cause patients to have initial worsening of their presenting symptoms, new-onset headache, or cranial neuropathy. This occurred in $23 \%$ of our patients, and mostly resolved with time, sometimes after a short steroid taper. Overall, these results suggest that flow diversion should be considered a viable treatment for symptomatic, cavernous ICA aneurysms in selected patients.

Traditional methods for treatment of cavernous sinus aneurysms, such as carotid sacrifice with or without bypass, have been very effective. Carotid sacrifice achieves a $98.7 \%$ rate of complete aneurysm occlusion and an $81 \%$ resolution of diplopia but is associated with a $5 \%$ risk of procedure-related neurologic deficits. ${ }^{4}$ Furthermore, carotid sacrifice may lead to changes in the hemodynamics of cerebral blood flow and increased stress on arterial walls of the contralateral carotid circulation..$^{714-20}$ This hemodynamic issue is of particular importance in patients with large, cavernous sinus aneurysms because they are often young females who can have an intrinsic weakness of the carotid vessel wall. ${ }^{21}$ This may predispose to the formation and growth of aneurysms contralateral to carotid sacrifice (estimated incidence, $4 \%-11 \%$ in a previous review $^{5}$ ) and also may hinder treatment choices of contralateral mirror aneurysms in the future. Unlike carotid sacrifice, endovascular coiling is a "reconstructive" technique that has been used in the treatment of cavernous sinus aneurysms. However, because these aneurysms are often very large or giant with a wide neck, standard endovascular coiling achieved a complete aneurysm occlusion rate of only $43 \%$ in a systematic review from $2002{ }^{4}$

Flow diversion may provide a valid alternative to these more traditional endovascular techniques by preserving the parent artery while achieving high complete occlusion rates. A recent publication of the results from the Pipeline for Uncoilable or Failed Aneurysms multicenter trial of flow diversion for intracranial aneurysms of the ICA proximal to the origin of the posterior communicating artery demonstrated an overall 5\% morbidity, similar to that in ICA sacrifice, though subset analysis of morbidity in patients treated for cavernous ICA aneurysms was not performed. ${ }^{9}$

Another review and meta-analysis of flow diversion demonstrated an all-comer late complication rate of $2.4 \% .^{22}$ Few studies have subset analyses specific to cavernous segment aneurysms, but in a multicenter Italian study, Briganti et $\mathrm{al}^{23}$ reported a $4 \%$ mortality rate for treatment of cavernous segment aneurysms with flow diverters, but this comprised 3 total cases (of 76): Two patients experienced fatal ICA thrombosis, and the other experienced a fatal ICA perforation, all during treatment of giant cavernous aneurysms. The mortality rate reported in the Italian study differs from that in both the subset analysis in the recent Canadian study $^{12}$ and in this series (both with $0 \%$ overall morbidity and mortality, 70 total combined cavernous aneurysms).

However, despite the very low incidence of permanent neurologic symptoms in patients in this series, technical complications were not uncommon. These were mostly related to vasospasm, incomplete device opening, and difficulty passing the catheter 
through the segment harboring the aneurysm. There were 4 instances of vessel perforation while attempting to obtain adequate catheter positioning for treatment. With further improvement of implant construction and delivery techniques, the incidence of these complications may decrease. Nevertheless, these issues underscore the fact that many of these procedures are not always smooth and straightforward. Keeping in mind these issues, we have slightly modified our approach, and after a phase of probably "overenthusiasm" for a novel technology, we now tend to be more cautious, especially in elderly patients. For elderly patients with challenging aneurysms, in whom issues with traversing the aneurysm or excessive proximal tortuosity can be anticipated, we include balloon test occlusion at the outset of the procedure, and in the presence of adequate collateral circulation, treatment via ICA sacrifice may be preferred.

This study is limited by its small sample size and the fact that multiple, different, flow-diverting stents were used, making it difficult to attribute these data to any particular device with its associated specifications. Timelines for radiographic follow-up were different between the 2 institutions, and not all patients in the cohort adhered to strict radiographic follow-up schedules, so it is difficult to determine which patients with incomplete occlusion at final follow-up in this series will eventually demonstrate complete occlusion. We did not perform any comparison of flow diversion with standard endovascular coiling techniques or carotid occlusion in this series; thus, we must be careful in comparing our results with those of other independent series. The patients in this cohort were highly selected, treated at 2 centers, both having very extensive experience with treatment of intracranial aneurysms; due to the relative novelty of flow diversion, the patients were carefully followed with adequate clinical and radiographic follow-up in nearly every case.

\section{CONCLUSIONS}

Flow-diverting stents represent a highly effective treatment technique for aneurysms of the cavernous ICA and may be a safer choice than carotid sacrifice with or without surgical bypass, given the ideal vessel characteristics of the cavernous ICA for flow diversion. Technical issues are not uncommon, occurring in just more than 1 in 3 cases, some of which were serious complications, though none resulted in any permanent neurologic morbidity in this series. Nevertheless, these technical issues, combined with the often advanced age of these patients and their relative vessel fragility, require a careful analysis to choose the procedure best indicated in each individual case.

Disclosures: Giuseppe Lanzino-UNRELATED: Board Membership: Edge Therapeutics, ${ }^{*}$ Consultancy: ev3/Covidien, ${ }^{*}$ Stryker. ${ }^{*}$ Luca Piero Valvassori-UNRELATED: Consultancy: Covidien, AB Medica, Travel/Accommodations/Meeting Expenses Unrelated to Activities Listed: Covidien, MicroVention, Other: Covidien/ev3, Comments: fees for participation in review activities such as data monitoring boards, statistical analysis, end point committees, and the like. David F. Kallmes-UNRELATED: Consultancy: ev3, ${ }^{*}$ Codman, ${ }^{*}$ Medtronic, ${ }^{\star}$ Comments: planning and implementation of clinical trials, Grants/Grants Pending: MicroVention, ${ }^{\star}$ ev3, ${ }^{*}$ Sequent, ${ }^{\star}$ Codman, ${ }^{*}$ Comments: preclinical and clinical research, Royalties: University of Virginia Patent Foundation, Comments: spine fusion. Harry J. Cloft—UNRELATED: Grants/ Grants Pending: Cordis Endovascular, ${ }^{*}$ Comments: Site principal investigator at enrolling site for Stenting and Angioplasty with Protection in Patients and Hlgh Risk for Endarterectomy registry sponsored by Cordis Endovascular. Edoardo BoccardiUNRELATED: Consultancy: Balt, Covidien. *Money paid to the institution.

\section{REFERENCES}

1. Kupersmith MJ, Stiebel-Kalish H, Huna-Baron R, et al. Cavernous carotid aneurysms rarely cause subarachnoid hemorrhage or major neurologic morbidity. J Stroke Cerebrovasc Dis 2002;11:9-14

2. Kupersmith MJ, Hurst R, Berenstein A, et al. The benign course of cavernous carotid artery aneurysms. J Neurosurg 1992;77:690-93

3. van Rooij WJ. Endovascular treatment of cavernous sinus aneurysms. AJNR Am J Neuroradiol 2012;33:323-26

4. van der Schaaf IC, Brilstra EH, Buskens E, et al. Endovascular treatment of aneurysms in the cavernous sinus: a systematic review on balloon occlusion of the parent vessel and embolization with coils. Stroke 2002;33:313-18

5. Dyste GN, Beck DW. De novo aneurysm formation following carotid ligation: case report and review of the literature. Neurosurgery 1989;24:88-92

6. Arambepola PK, McEvoy SD, Bulsara KR. De novo aneurysm formation after carotid artery occlusion for cerebral aneurysms. Skull Base 2010;20:405-08

7. Tomsick T. Long-term clinical follow-up of therapeutic internal carotid artery occlusion. AJNR Am J Neuroradiol 2007;28:1626

8. Arnaout OM, Rahme RJ, Aoun SG, et al. De novo large fusiform posterior circulation intracranial aneurysm presenting with subarachnoid hemorrhage 7 years after therapeutic internal carotid artery occlusion: case report and review of the literature. Neurosurgery 2012;71:E764-71

9. Becske T, Kallmes DF, Saatci I, et al. Pipeline for uncoilable or failed aneurysms: results from a multicenter clinical trial. Radiology 2013;267:858-68

10. Piano M, Valvassori L, Quilici L, et al. Midterm and long-term follow-up of cerebral aneurysms treated with flow diverter devices: a single-center experience. J Neurosurg 2013;118:408-16

11. Lanzino G. Editorial: ophthalmic segment aneurysms. J Neurosurg 2013;118:935, discussion 935-36

12. O'Kelly CJ, Spears J, Chow M, et al. Canadian experience with the pipeline embolization device for repair of unruptured intracranial aneurysms. AJNR Am J Neuroradiol 2013;34:381-87

13. Szikora I, Marosfoi M, Salomvary B, et al. Resolution of mass effect and compression symptoms following endoluminal flow diversion for the treatment of intracranial aneurysms. AJNR Am J Neuroradiol 2013;34:935-39

14. Gurdjian ES, Lindner DW, Thomas LM. Experiences with ligation of the common carotid artery for treatment of aneurysms of the internal carotid artery. J Neurosurg 1965;23:311-18

15. Drapkin AJ, Rose WS. Serial development of 'de novo' aneurysms after carotid ligation: case report. Surg Neurol 1992;38:302-08

16. Klemme WM. Hemorrhage from a previously undemonstrated intracranial aneurysm as a late complication of carotid artery ligation: case report. J Neurosurg 1977;46:654-58

17. Niiro M, Shimozuru T, Nakamura K, et al. Long-term follow-up study of patients with cavernous sinus aneurysm treated by proximal occlusion. Neurol Med Chir (Tokyo) 2000;40:88-96, discussion 96-87

18. Ostergaard JR. A long-term follow-up study of juvenile aneurysm patients. Acta Neurochir (Wien) 1985;77:103-09

19. Parekh HC, Prabhu SS, Keogh AJ. De novo development of saccular aneurysms: report of two cases. Br J Neurosurg 1995;9:695-98

20. Winn HR, Richardson AE, Jane JA. Late morbidity and mortality of common carotid ligation for posterior communicating aneurysms: a comparison to conservative treatment. J Neurosurg 1977;47:727-36

21. Brisman JL, Song JK, Newell DW. Cerebral aneurysms. N Engl J Med 2006;355:928-39

22. Arrese I, Sarabia R, Pintado R, et al. Flow-diverter devices for intracranial aneurysms: systematic review and meta-analysis. Neurosurgery 2013;73:193-99, discussion 199-200

23. Briganti F, Napoli M, Tortora F, et al. Italian multicenter experience with flow-diverter devices for intracranial unruptured aneurysm treatment with periprocedural complications: a retrospective data analysis. Neuroradiology 2012;54:1145-52 